\title{
Re-Visiting the Anisotropy of Inertia Experiments
}

\author{
Robert L. Shuler \\ NASA Johnson Space Center/EV5, 2101 NASA Parkway, Houston, TX 77058 \\ Email: robert.1.shuler@nasa.gov
}

\begin{abstract}
In the 1960s experiments investigating anisotropy of inertia relative to solar or galactic mass centers using the Mössbauer effect obtained negative results. Both sides of a debate over Mach's Principle claimed the result was what should be expected. However in light of earlier comments by Einstein on the relativity of inertia to masses, Brans and Dicke felt a revised theory of gravity would better incorporate Mach's Principle. We present a new view that the old experiment assumed, incorrectly, that Mach's Principle affects only time dilation, which would violate the Equivalence Principle, and that the results were a predictable coordinate artifact. Using a special formalism of Distant Inertial and Spatially Homogeneous coordinates we give a plausible analysis that radial spatial distortion in a gravitational field is also related to Mach's Principle and embodies the expected anisotropy while keeping equivalence locally intact. This leads to a view of momentum interactions via the space-time field that invites further analysis. Also, since Mach's principle seems to be related to both time and spatial curvature, we briefly discuss whether it could be used as a postulate basis for space-time and how this might affect experiments designed to detect or exclude matter-coupled fields.
\end{abstract}

Keywords: Gravity; general relativity; space-time; equivalence; Mach's principle

\section{Introduction}

Einstein did not base General Relativity (GR) on Mach's Principle, a term he coined, but stated he was influenced by it and expected it to emerge in the final theory. In 1912 he derived using Newtonian gravity and Special Relativity (SR) a general relation for the contribution to inertia of a mass shell $M$ of radius $r$ to a mass $m$ in its center of $\Delta m / m=G M / r c^{2}$ [1]. Of course the contribution from a spherical shell will not manifest isotropy. Lacking anisotropy, and based on gravity which has universal effect on all mass-energy, it is clear that such changes in inertia are not detectable. They affect all local clocks by which acceleration and thereby inertia is measured.

By 1917 Einstein was convinced of success, stating: "In a consistent theory of relativity there can be no inertia relatively to space, but only an inertia of masses relatively to one another. If, therefore, I remove a mass to a sufficient distance from all other masses in the universe, its inertia must fall to zero,"[2] a position that shortly came under criticism by noting a mass-less solution to the field equations was possible in which an infinitesimal test mass was found to have inertia. We introduce this argument only to dismiss it as semantic, since the "removed" test mass in the case Einstein cited indeed has mass relative to infinitely fast local test clocks. But for field theory reasons Einstein later reversed his position. He did not provide details, but we suspect it has to do with the form of the vacuum equation and the locality of curvature generation. The 1912 formulation implies that inertia due to a summation over all such shells[3] reduces to a coefficient of "1," and thus an approximation for the inertia modification from a nearby mass $M$ is $m^{\prime}=m\left(1+G M / r c^{2}\right)$. While approximately the same as the Schwarzschild time dilation factor $\left(1-2 G M / r c^{2}\right)^{-1 / 2}$, at very small $r$ it is too weak to account for curvature that must appear, violating either the vacuum equation or more likely the direct local generation of space-time curvature by sources and requiring an intermediary field, a complexity Einstein did not want. For Einstein space-time is the field and the only field accounting for gravity.

In 1958 Cocconi and Salpeter suggested the possibility that inertial relativity might have a vector dependency, resulting in anisotropy due to non-spherical shell mass distributions such as the nearby Sun or Milky Way[4]. This effect would not be rendered immeasurable by clocks since it would vary with 
orientation. Use of the Mössbauer effect to test this hypothesis was suggested[5] and almost immediately measurements were made and a limit for anisotropy was obtained as $\Delta m / m \leq 5 \times 10^{-23}[6]$. A further discussion of the history of this formulation of inertia, including a controversial radiation reaction version found by Sciama who was unaware of Einstein 1912, and the splitting of opinion following the anisotropy tests as to whether this finding was in conflict with Mach's Principle or ultimately predicted by the EP from which the 1912 formulation of Mach's Principle can also be derived, is found in a previous paper[7].

The dilemma can be summed up as follows. It seems that acceleration which does not change the distance to the inertia-causing mass (e.g. tangential) should have no or less effect than acceleration toward or away from the inertia-causing mass (radial), producing a conflict with the EP. Yet Mach's Principle can be derived from the EP (Ibid. [7]) and must be compatible with it. Note that the EP is generally considered inadequate for describing the anisotropic aspect of the Schwarzschild metric: spatial expansion[8] (applying only radially, not tangentially), and it is there we look for evidence of anisotropy which does not violate equivalence. First we develop a coordinate formalism with sufficient physical characteristics to find anisotropy (since local tangent Minkowski space rules it out), and develop an understanding of the spatial anisotropy that exists within GR. Then we discuss briefly some possible characteristics of an intermediate field that would bridge the gap between GR and Mach's Principle in the strong field regime where $\left(1-2 G M / r c^{2}\right)^{-1 / 2}>\left(1+G M / r c^{2}\right)$ and how this relates to potential future Astrophysical experiments.

\section{Method}

\subsection{Distant Inertial and Spatially Homogeneous (DISH) Coordinates}

Local Inertial Frames (LIFs) in GR are considered to be small Minkowski tangent spaces. Within them coordinates can be chosen which have physical meaning as proper times and Euclidean distances, and physical laws can be expressed in ordinary Newtonian or SR forms depending on velocity, but the integration of such proper measures over changing spaces rapidly loses significance for expressing simple, clear physical laws. We also know from Noether's theorem, for example, that the useful principle of conservation of momentum depends on homogeneity of space, and conservation of energy on homogeneity of time[9]. Since conservation of momentum is essentially an expression of inertia, we find it simpler to have homogeneous spatial coordinates over the region, small or large, within which we wish to formulate or measure inertia.

Consider a tangent Minkowski space D extended over astronomical distances for which it yet provides a close approximation to local tangent spaces where no matter is near. It still may be considered "small" and almost differential volume by cosmological or even galactic standards, and may even have gravitational objects (stars, pulsars, black holes) embedded in it which when not interacting may for practical purposes be treated like points. Light traveling directly away from such objects, not passing near other similar objects, may be considered to follow a straight path and used to triangulate position of the nearly point-like objects in a Euclidean coordinate grid. Black holes can be located similarly using either their star shadows or light emitted when material is absorbed. We will call these Distant Inertial and Spatially Homogeneous (DISH) coordinates.

Near to each point-like gravitational object, where its field no longer appears point-like, Schwarzschild $r=C / 2 \pi$ spherical coordinates can be used to assign Euclidean coordinate designations arbitrarily to points within the field. As long as such a point is not moved relative to the nearby mass that defines its local space-time, then as the nearby mass moves through a proper distance in $\mathrm{D}$, so does the point. Small tangential distances will correspond to DISH coordinate distances, while radial distances in proper units will be greater than the measure between the same two points located in the coordinate space and measured with DISH units which otherwise (away from gravitating objects) correspond to proper distances. If the near-object metric is $d s^{2}=-d \tau^{2} c^{2}=-\Upsilon^{-2} c^{2} d t^{2}+\Upsilon^{2} d r^{2}+r^{2}\left(d \theta^{2}+\sin ^{2} \theta d \phi^{2}\right)$ where $\Upsilon=\left(1-2 G M / r c^{2}\right)^{-1 / 2}$ and an object (matter or photon) is moving with radial and tangential component velocities $\mathbf{v}=\mathbf{v}_{t}+\mathbf{v}_{r}$ then the coordinate velocity will be $\mathbf{v}_{\text {DISH }}=\left(\mathbf{v}_{t}+\mathbf{v}_{r} \Upsilon^{-1}\right) \Upsilon^{-1}$.

At large distances from the mass in what one might call distant observer coordinates, the Schwarzschild metric becomes asymptotically congruent with D. Ordinary Newtonian and SR laws will 
hold in these coordinates as long as both time and spatial resolutions make the gravitating objects and interactions with them of small spatial-temporal extent compared to measurements and calculations. For example, if object of momentum $m \mathbf{v}$ collides with black hole of mass $M$ which was for convenience previously considered at rest in $\mathrm{D}$, then the resultant velocity of $M$ is $\mathbf{v}^{\prime}=m \mathbf{v} /(m+M)$, even though in $\mathrm{D}$ observer time the object $m$ never actually contacts $M$ but only reaches the event horizon where its coordinate velocity vanishes. This suggests, though we do not depend upon or attempt to prove, that at all times the center of mass of $m$ and $M$ is moving at $\mathbf{v}^{\prime}$.

Already we have some insight into relative or "Machian" inertia. The total inertia $m+M$ does not change with proximity, only the ability to move $m$ relative to $M$ changes with proximity, with $m$ finally getting stuck on the event horizon if $M$ is a black hole.

\section{$2.2 \quad$ Two Measures of Relative Inertia}

With this choice of coordinates, which are nonetheless similar in many respects to an ordinary inertial reference frame with proper distance coordinates, we see almost trivially that there are two measures of "stickiness" or relative inertia of $m$ with respect to $M$. The first is isotropic in both local coordinates and DISH coordinates, the slowing of clocks of all types regardless of their axes of motion due to the time dilation effect on coordinate velocity given by $\Upsilon=\left(1-2 G M / r c^{2}\right)^{-1 / 2} \approx 1+G M / r c^{2}$, which fully accounts for the old 1912 formulation of Machian inertia and all that can be derived from equivalence.

A second factor equal in magnitude, but directionally sensitive to the radial axis as Cocconi and Salpeter suspected, is due to the radial spatial coefficient. But since it affects both coordinate velocity and the distances through which objects or energy travel, it does not affect the timing of clocks. It does, however, affect the DISH coordinate distance through which moving objects can be displaced with a given amount of work, or their resistance to displacement relative to $M$ (aka relative inertia). So by following the equations of motion in a Schwarzschild metric, we conclude that there are two Mach-like effects of approximately the correct magnitude, one which is isotropic and affects coordinate time, and one which is anisotropic and affects coordinate distance.

Since the radial inertia is vector-like, for the mass-shell where all directions are present the anisotropy entirely cancels out. Thus the necessity of considering it didn't arise in cosmological treatments of inertia (e.g. [10]) where uniform isotropic distributions of contributing mass were assumed. In general a relativistically consistent treatment of a finite set of masses will not be obtainable because of the nointeraction theorem[11]. This should not be a problem since the results were derived from the space-time field of GR, but having treated two sources as point-like to make the momentum and inertial effects clear, it was felt advisable to mention that this point-like treatment cannot be easily extended.

\subsection{The Possibility of Purely Machian Inertia and Emergent Gravity}

As far as approximately Machian inertia and explaining the negative result of the anisotropy experiments, entirely within GR, we are done. It is not essential to go further. But just as curiosity about a radial component to Machian inertia stimulated curiosity to conduct experiments and eventually to explain them in the current paper, providing new and unexpected insight into the components of relative inertia and its relation to equivalence, one may well wonder why the Machian proximity relation does not hold as well for strong fields, resulting in time dilation and spatial expansion factors with $\Upsilon=1+G M / r c^{2}$ exactly.

Considering that the inverse of time, i.e. frequency, may be equated with energy via the Planck relation, then an ordinary Hamiltonian or Lagrangian formulation with the Machian relative inertia postulated to provide time dilation provides the correct gravitational acceleration as an emergent property. This is true as far as we know from experiment, since actual field strength in the $2^{\text {nd }}$ order regime where $\left(1-2 G M / r c^{2}\right)^{-1 / 2}>\left(1+G M / r c^{2}\right)$ has not been verified. (Higher order verification in $v / c$ using Pulsars is done using linearized GR for gravitational wave losses[12], not $2^{\text {nd }}$ order field strength.)

If we further postulate the radial component of relative inertia, then by maintaining the requirement for the EP to hold we obtain the requirement for isotropic clock timing, and can reverse the arguments of the previous section to obtain the radial space expansion factor.

The "problem" with this reasoning is that either the vacuum equation or the source term of GR would have to give way in strong fields, and it is hard to do either consistently. The vacuum equation 
simply redistributes the generated curvature over a larger 4-surface, so that curvature, or the source, is "conserved" as one popular text puts it[13]. Some texts draw an analogy to the Newtonian Poisson equation[14][15]. If the near field is too weak, then there is not a plausible way to restore the correct amount of curvature for the far field where both GR and Machian theories agree the vacuum equation is correct.

A solution for some issues in GR is to assume some particular type or distribution of the mass-energy source term, such as dark matter, dark energy, etc. In this case however, we have to assume a redistribution of the effectiveness of the source term in generating curvature over a larger volume than is actually occupied by the source. To avoid non-locality, this requires an intermediate field. The source must generate a $1 / r$ potential field which in turn generates the correct amount of curvature at some distance from the source.

We have two precedents that the "mass" effect of matter-energy is not precisely and entirely at the location of the matter-energy. One is the momentum interaction we examined in section 2.1. Depending on how close $m$ and $M$ are, pushing on one to some degree pushes the other even though they are not in physical contact. This effect is, however, entirely a consequence of the temporal and spatial coefficients, and should not be used to explain them. The other precedent is the uncertainty principle, by which the position and momentum (mass effect) of a differential source element (mass) cannot both be known exactly. The quantum state function of momentum is three orthogonal state functions each proportional to $1 / r$ across the Heisenberg uncertainty inequality. The magnitude of the superposition of these functions therefore has the desired $1 / r$ proportionality. To develop this first factor, the mass from the uncertain quantities in the uncertainty relation is used along a single dimension: $\Delta x \Delta \rho \geq \hbar / 2 \Leftrightarrow$ $\Delta x \cdot m \Delta v \geq \hbar / 2$. Next assume an average velocity uncertainty over some measurement ensemble and incorporate this into a factor $k$ to get rid of it: $m \approx \hbar / 2 \Delta v_{\text {avg }} \Delta x \Leftrightarrow m \approx k / \Delta x$. Then realize that by reorienting the $x, y$ and $z$ axes we can always have one axis in an arbitrary radial direction $\Delta x=r$, and calibrate $k$ using the classical arguments from any of the Machian inertia derivations summed over all contributing mass elements and we have inertial mass $m^{\prime}$ relative to the inertia-causing mass-energy $m$ as $m^{\prime} / m=1 \approx \int_{i} G M_{i} / r_{i} c^{2}$.

Distant matter is relatively more important since equal thickness mass shells, assuming reasonably uniform distribution of matter, increase in volume faster than their contribution due to distance declines. Subtracting out one large nearby mass $M$ we still have approximately " 1 " left in the cosmological inertia integral. Thus the local inertia relative to $M$ is $m^{\prime}=m\left(1+G M / r c^{2}\right)$. This gives the required time dilation factor for emergent gravity and suggests curvature is due to the potential term $G M / r c^{2}$. The details of writing a dynamic field equation incorporating this intermediate field as the curvature generator are beyond the scope of this paper, but we suppose there is a good chance they would result in a theory of gravity similar to the one we have, but differentiated in extremes of strong fields and also possibly at cosmological distances.

\subsection{Relation of Gravity-Based Inertia Formulations to Quantum Theories of Inertia}

We shall briefly and qualitatively discuss how gravitational formulations of inertia do and don't fit in with two well-known quantum theories related to inertia. First, the well-known Higgs field, recently confirmed through the discovery of the Higgs boson[16], gives mass to massive elementary particles in electroweak symmetry breaking by means of a scalar particle, the Higgs boson. This particle has mass in the range of 124-126 GeV. The Higgs field effectively attaches its pre-existing mass-energy (inertia) to the particles which are massive after symmetry breaking. In other words, the Higgs mechanism does not formulate a mechanism of inertia generally, but reallocates (one might say) existing inertia, and the way it would add to the reader's inertia is to carry a backpack full of lead weights. There is little intersection of, and no conflict between, our discussion and the Higgs mechanism.

A more complex relation exists to the idea of inertia as induced by the Zero-Point Field (ZPF), which has been evolving over several decades as a relatively complex mechanism, the mechanics and bases of which are still being evolved[17][18], and finally connected (somewhat) with gravity[19]. The ZPF refers to the electromagnetic (or the QED) quantum vacuum, which differs in theoretical value from the gravitational vacuum energy by one of the largest ratios in all of physics. This has not been addressed 
by ZPF advocates. One could, presumably, just assume some scale factor, but as the ZPF community turns toward experiments, they are only at the point of asking how much of inertia is even due to the ZPF, while for our purposes we are talking unconditionally about "all" of it. For purposes of continuing the discussion, let us assume that eventually the ZPF will be shown to be proportional to gravitational potential, probably with gauge factors involved, and without assurance this result will actually be achieved. Then the local inertia could be satisfactorily explained in a classical and quantum duality (gravity and Mach's principle, and the presumably related ZPF).

This exposes a second difficulty. Quantum mechanics is formulated usually in local reference frames, and would not expose the type of asymmetry we have been discussing (distortion of the radial proper distances in distant homogeneous coordinates), unless specifically formulated to do so. It is not exposed directly by experiment anymore than the measurements of inertia asymmetry in the Mössbauer effect, which showed up not as clock rate changes but as clock displacement changes, again only with reference to momentum-preserving global coordinates where the displacement might be compared with the displacement of a distant clock under momentum (inertia)-preserving conditions.

The author wishes to point out that if a complete explanation of inertia from quantum phenomena is achieved, which matches the gravitational changes to time and space without assuming them, then effectively a constructive theory of relativistic gravity is obtained (because inertial motion, including light bending and the fall of objects, would result from geodesics in the implicitly constructed space based on the time dilation and spatial displacement changes derived from the inertia theory). This is not impossible, but difficult for physicists to imagine at present, and might not have the cosmological features of GR, nor the unobservable features (beyond event horizons).

On the other hand, if a quantum theory of inertia is developed which works assuming the space-times of GR, then it already incorporates gravity, and by inference inertia at least in the classical sense. This would still be quite useful, but it would add essentially nothing to the theory of gravity, merely exchanging the puzzle of the relation of inertia and curved space-time for the puzzle of the relation of the ZPF to curved space-time.

\section{$3 \quad$ Discussion of Results}

The immediate result is two-fold. First a satisfactory explanation of an old experiment shows that according to GR, when spatial factors are considered, something which can be interpreted as an anisotropic component of relative inertia is present, but it does not affect clocks. Second, the magnitude is the same as time dilation related inertia, but applies only in the radial direction. We considered only static symmetric fields, with the aid of Distant Inertial and Spatially Homogeneous coordinates (similar to distant observer coordinates), and found other interesting results with regard to momentum coupling through the gravitational field.

We found that approximate Machian inertia is contained within GR, but that formulating relative inertia as a postulate causes difficulty with the field equation. This difficulty can probably be resolved by using an intermediate potential field to generate curvature. The effect is to change predictions of properties of astrophysical objects. In the extreme case of a purely Machian emergent gravity, an event horizon would not form, though certainly extremely dense massive objects would still form. Neutron stars about 6 to 10 times the radius of a black hole of similar mass would be little affected. If there is even a partial effect of uncertainty of position or other quantum constraint on the distribution of curvature-causing source distribution, whether that is the mass-energy itself, or the probability of interacting with it, then the requirements for complete collapse would be increased. This should result in a greater variety of states of mass-energy that might eventually be observable in near-collapse objects of insufficient mass for full collapse. Since a similar field equation would be used, with the added intermediate field having effect mostly in strong-field short-range situations, the dynamics of gravitational waves and most currently observable phenomena would not be affected.

Figure 1 shows comparative light bending, calculated with a numerical technique for finding geodesics in an arbitrary metric, for a 4 solar mass object at various radii. Also shown for reference is a first order bending analytic calculation using the method given by Bodenner and Will[20]. While it is doubtful the parameters for mass, gravitational constant and so forth can be determined with sufficient accuracy to discriminate these two cases for a single measurement point, the overall shape of the curves appear to 
provide discrimination, provided the object is less than about $50 \mathrm{~km}$ in radius. Interferometry in the microarcsecond range may be possible[21], but only has the ability for kilometer resolution about a lightyear. We can either wait for further improvements, or launch interferometer clusters into deep space to take advantage of the sun as a gravitational lens[22]. The uses of such an instrument would certainly not be limited to tests of GR, and quite probably the primary goal would be observations of exoplanets at high resolution.

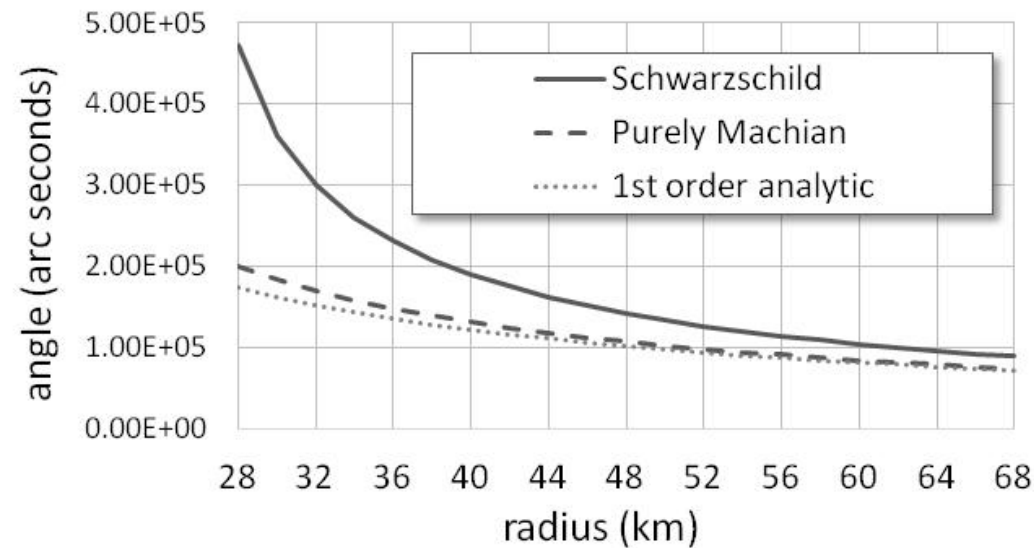

Figure 1. Light bending for Machian vs Schwarzschild strong fields near $4 M_{\odot}$ object

The ease with which nature "covered up" a potential equivalence violation, distorting space to do so it would seem in retrospect, is unsettling. There is some interest in a variety of possible fields which couple directly to matter and are associated with advanced quantum theories[23]. By the reversal of arguments described in section 2.3, equivalence becomes a primary postulate for any universally coupled field and we can imply curvature as necessary to enforce it. This seems to have not been previously realized because potentially equivalence violating effects, such as anisotropic inertia, were presumed not to exist as a result of negative test outcomes. We have shown anisotropic inertia does exist, and that space-time curvature conceals it, upholding equivalence, making its apparent non-existence an artifact of the choice of local Minkowski (equivalence) space for measurement. It happens that spatial curvature was already predicted by GR, but we would be naïve to think a similar scenario could not play out for other fields. Anything that universally couples to matter-energy and has a work function must generate time dilation to accommodate energy conservation, and may generate spatial curvature as well. This will be explored in a separate paper on a strong field verification framework.

\section{Conclusion}

A better understanding of the relation between equivalence and spatial curvature was unexpectedly obtained from our investigation of an old experiment. In addition, a system of coordinates for measurement of proper quantities over astronomical distances containing embedded objects with strong local curvature makes it possible to talk about things like conservation of momentum of propagating black holes with greater clarity. Machian concepts are more valid in GR than previously realized, but a purely Machian formulation has difficulty in the region of strong fields. A purely Machian formulation, though speculative, provides a very close approximation to GR and a possible reference target to guide GR verification experiments. It is also possible to find quantum principles that justify the required $1 / r$ proportional intermediate field, which might be fertile grounds for quantum gravity investigations. Experiments to detect or exclude coupled fields must be carefully designed to avoid misinterpretation similar to what apparently occurred with anisotropy of inertia.

\section{References}

1. Einstein, "Is There a Gravitational Effect Which Is Analogous to Electrodynamic Induction?" Vierteljahrschrift für gerichtliche Medizin (ser. 3), 44, 37-40 (1912). Available in The Collected Papers of Albert Einstein, Vol. 4 (English), trans. Anna Beck, Princeton University Press, Princeton (1996). 
2. A. Einstein, in Mach's Principle: From Newton's Bucket to Quantum Gravity, edited by J. Barbour and H. Pfister, Birkhauser, Basel, Switzerland, (1995).

3. R.L. Shuler, "A Fresh Spin on Newton's Bucket,' Phys. Ed., 50, 1 p88 (2015).

4. G. Cocconi and E. E. Salpeter, "A Search for Anisotropy of Inertia," Nuovo Cimento, 10, 646-651 (1958).

5. G. Cocconi and E. E. Salpeter, "Upper Limit for the Anisotropy of Inertia from the Mössbauer Effect," Phys. Rev. Letters, 4, 176-177 (1960).

6. R. W. P. Drever, "A Search for Anisotropy of Inertial Mass using a Free Precession Technique," Phil. Mag., 6, 683-687 (1961).

7. R. Shuler, "Isotropy, Equivalence and the Laws of Inertia," Phys. Essays, 24, 4 (2011) pp. 498-507.

8. R.P. Gruber, R.H. Price, S.M. Matthews, W.R. Cordwell, and L.F. Wagner, "The Impossibility of a Simple Derivation of the Schwarzschild Metric," Am. J. Phys., 56, 265-269 (1988).

9. J. Hanc, S. Tuleja and M. Hancova, "Symmetries and Conservation Laws: Consequences of Noether's Theorem," Am. J. Phys., 72, 4, pp 428-435 (2004).

10. I. Ciufolini and J. Wheeler, Gravitation and Inertia, Princeton University Press, Princeton, New Jersey (1995).

11. H. Leutwyler, "A No-Interaction Theorem in Classical Relativistic Hamiltonian Particle Mechanics," Nuovo Cimento, 37, 2, 556-567 (1965).

12. J.H. Taylor, L.A. Fowler and P.M. McCulloch, "Measurements of General Relativistic Effects in the Binary Pulsar PSR1913+16," Nature, 277, 8, pp. 437-440 (1979).

13. C.W. Misner, K.S. Thorne and J.A. Wheeler, Gravitation, W.H. Freeman \& Co., San Francisco (1973) p 429.

14. M. Blau, "Lecture Notes on General Relativity," Institute for Theoretical Physics, University of Bern, Switzerland (2014). http://www.blau.itp.unibe.ch/Lecturenotes.html15. National Center for Biotechnology Information, Available: http://www.ncbi.nlm.nih.gov

15. W. Rindler, Relativity Special, General, and Cosmological, 2nd ed., Oxford University Press, Oxford - New York, p. 221 (2006).

16. G. Aad et. al., "Observation of a New Particle in the Search for the Standard Model Higgs Boson with the ATLAS Detector at the LHC," Phys. Lett. B, 716, 1, pp 1-29 (2012).

17. B. Haisch, A. Rueda and H.E. Puthoff, "Inertia as a Zero-Point Lorentz Force," Phys. Rev. A, 49, 2, pp 678-694 (1994).

18. B. Haisch, A. Rueda and Y. Dobyns, "Inertial Mass and the Quantum Vacuum Fields," Ann. Phys., 10, 5, pp292-414 (2001).

19. A. Rueda, B. Haisch, "Gravity and the Quantum Vacuum Inertia Hypothesis," Ann. Phys., 14, 8, pp. 479-498 (2005).

20. J. Bodenner and C. Will., "Deflection of Light to Second Order: A Tool for Illustrating Principles of General Relativity," Am. J. Phys., 71, 8 (2003).

21. Kopeikin, S.M. and Makarov, V.V., "Gravitational Bending of Light by Planetary Multipoles and its Measurement with Microarcsecond Astronomical Interferometers," Phys. Rev. D, 75, 6, p. 062002 (2007).

22. Maccone, C., "Space Missions Outside the Solar System to Exploit the Gravitational Lens of the Sun," J. Brit. Interplanetary Soc., 47, 2, pp. 45-52 (1994).

23. D.F. Mota and D.J. Shaw, "Evading Equivalence Principle Violations, Cosmological and other Experimental Constraints in Scalar Field Theories with a Strong Coupling to Matter," Phys. Rev. D, 75, 063501 (2007). 